\title{
Dynamic Beamforming for Three-Dimensional MIMO Technique in LTE-Advanced Networks
}

\author{
Yan Li, Xiaodong Ji, Dong Liang, and Yuan Li \\ Wireless Signal Processing and Network Lab, Key Laboratory of Universal Wireless Communications (Ministry of Education), \\ Beijing University of Posts and Telecommunications, Beijing 100876, China
}

Correspondence should be addressed to Yan Li; ly19880928@gmail.com

Received 8 March 2013; Revised 3 July 2013; Accepted 4 July 2013

Academic Editor: Feifei Gao

Copyright (C) 2013 Yan Li et al. This is an open access article distributed under the Creative Commons Attribution License, which permits unrestricted use, distribution, and reproduction in any medium, provided the original work is properly cited.

\begin{abstract}
MIMO system with large number of antennas, referred to as large MIMO or massive MIMO, has drawn increased attention as they enable significant throughput and coverage improvement in LTE-Advanced networks. However, deploying huge number of antennas in both transmitters and receivers was a great challenge in the past few years. Three-dimensional MIMO (3D MIMO) is introduced as a promising technique in massive MIMO networks to enhance the cellular performance by deploying antenna elements in both horizontal and vertical dimensions. Radio propagation of user equipments (UE) is considered only in horizontal domain by applying $2 \mathrm{D}$ beamforming. In this paper, a dynamic beamforming algorithm is proposed where vertical domain of antenna is fully considered and beamforming vector can be obtained according to UEs' horizontal and vertical directions. Compared with the conventional 2D beamforming algorithm, throughput of cell edge UEs and cell center UEs can be improved by the proposed algorithm. System level simulation is performed to evaluate the proposed algorithm. In addition, the impacts of downtilt and intersite distance (ISD) on spectral efficiency and cell coverage are explored.
\end{abstract}

\section{Introduction}

As is generally known, long-term evolution (LTE) designed by the third generation partnership project (3GPP) helps operators to provide wireless broadband services with enhanced performance and capacity. A variety of targets and requirements for LTE have been suggested by 3GPP, including higher peak data rates and more UEs per cell as well as lower control plane latency than currently employed $3 \mathrm{G}$ architectures $[1,2]$. Based on orthogonal frequency division multiple access (OFDMA), radio technology applies various scheduling and multiantenna methods. 3G LTE is further developed to meet requirements set for IMT-Advanced technologies. The role of antenna parameter selection in evolution of 3G LTE-Advanced has been widely discussed currently [3].

As one of the key techniques in LTE-Advanced system, multiple input multiple output (MIMO) [4-6] is considered as an effective way to obtain high data rates without sacrificing bandwidth and to improve the performance of both cell edge UE and the whole system. A trend toward deploying larger number of antennas $[7,8]$ can be noted in the evolution of some standards such as IEEE 802.11n/802.11ac and LTE. With Massive MIMO, huge numbers of elements in antenna arrays are able to deployed in systems being built today [9]. Larger numbers of terminals can always be accommodated by combining massive MIMO technology with conventional time and frequency division multiplexing via orthogonal frequency division multiplexing (OFDM). Massive MIMO is a new research field in communication theory, propagation, and electronics and represents a paradigm shift in the way of considering with regard to theory, systems, and implementation.

Three-dimensional MIMO (3D MIMO) can be seen as an effective method to approach massive MIMO without applying too much antennas on transmitter or receiver. Vertical dimension will be utilized in the antenna modeling, and downtilt of the antennas will become significant channel parameters. A typical two-dimensional (2D) antenna is used to cover a sector of 120 degrees only in horizontal domain [10]. Compared with the 2D channel propagation, scatterer is no longer located in the same plane with antennas and is supposed to distribute randomly in three-dimensional space. 
We are considering the possibility of extending the current $2 \mathrm{D}$ antenna to the future $3 \mathrm{D}$ antenna, which means that the departure and arrival angles have to be modeled in two directions, that is, horizontal and vertical [11-13].

Beamforming of linear array antenna elements merely in horizontal dimension does not give full free-space gain. This is due to the azimuth spread of the received signal as seen from the base station (BS), which has been extensively investigated in previous studies. It is necessary to propose a novel beamforming algorithm which includes the gain of the vertical dimension. In this paper, we will present a dynamic beamforming algorithm in which vertical difference of $3 \mathrm{D}$ antennas will be taken into account. In conventional 2D MIMO scenario, cell edge UEs suffer serious intercell interference from neighboring cell due to their locations [14, $15]$. Since the radio propagation from a transmission node to a UE is divided into horizontal direction and vertical direction, the power of intercell interference can be reduced largely, which results in an enhancement of the signal to interference plus noise ratio (SINR). Compared with the existing 2D beamforming algorithm, better system performance can be brought by dynamic beamforming algorithm.

The rest of this paper is organized as follows. Section 2 describes the system model of the downlink LTE-Advanced networks and the modeling of 3D MIMO channel. The principle and details of the dynamic beamforming algorithm are introduced in Section 3, and performance of the proposed algorithm with different configuration parameters is simulated and analyzed in Section 4. The conclusion of this paper is given in Section 5 .

\section{System Model}

2.1. 3D MIMO System. The downlink of a cellular network with $M$ hexagonal cells is considered as depicted in Figure 1, and each cell is partitioned into 3 sectors with $K$ active UEs served within the coverage of each sector. The total number of BSs in the system is $3 M$. Each BS which corresponds to one sector is equipped with $N_{t}$ transmitting antennas, while each UE has $N_{r}$ receiving antennas. For the received signal at the $k$ th UE served by BS $m$, we have

$$
\begin{aligned}
\mathbf{y}_{m}^{(k)}= & \underbrace{\mathbf{H}_{m}^{(k)} \mathbf{W}_{m}^{(k)} \mathbf{s}_{m}^{(k)}}_{\text {desired signal }} \\
& +\underbrace{\sum_{n \neq m}^{3 M} \sum_{w=1}^{K} \mathbf{H}_{n}^{(k)} \mathbf{W}_{n}^{(k)} \mathbf{s}_{n}^{(w)}}_{\text {inter-sector interference }}+\mathbf{n}_{m}^{(k)},
\end{aligned}
$$

where $\mathbf{s}_{m}^{(k)}$ is the $l_{k} \times 1$ transmitted vector for UE $k$ served by BS $m$ and $l_{k}$ is the number of data stream. Denote $\mathbf{s}_{m}^{(k)}=$ $\left[\sqrt{p_{k}} s_{m, 1}^{(k)}, \sqrt{p_{k}} s_{m, 2}^{(k)}, \ldots, \sqrt{p_{k}} s_{m, l_{k}}^{(k)}\right]^{T}$, and $p_{k}$ is the transmitting power of each data stream at UE $k$. For 3D MIMO, due to the introduction of vertical dimension in transmitting antenna, the channel coefficient matrix is three-dimensional, that is, the number of receiving antenna, the number of transmitting antenna, and the number of elevation element. It is assumed that each transmitting antenna has $N_{v}$ elements,

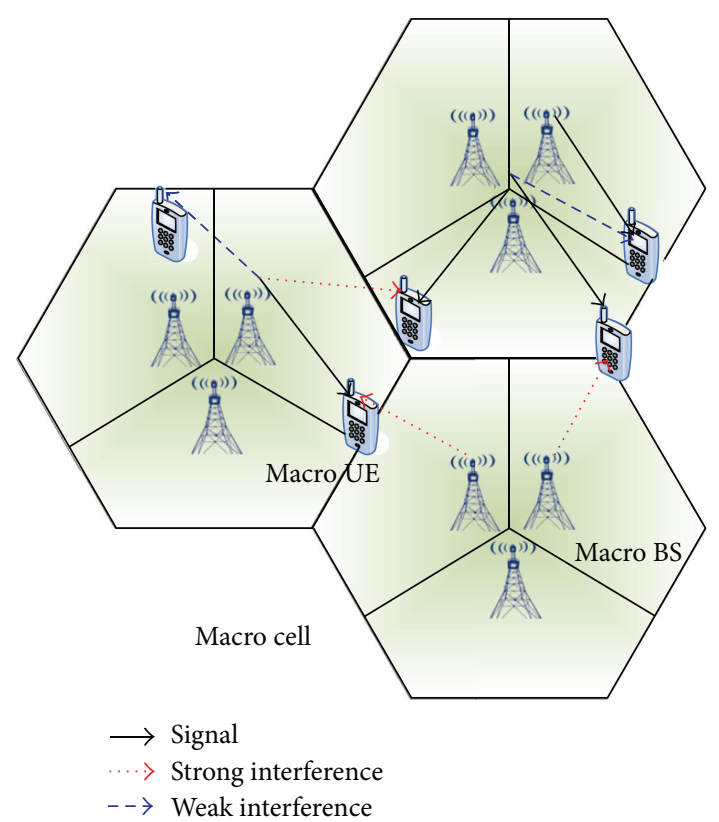

FIGURE 1: System model for downlink transmission of LTEAdvanced networks.

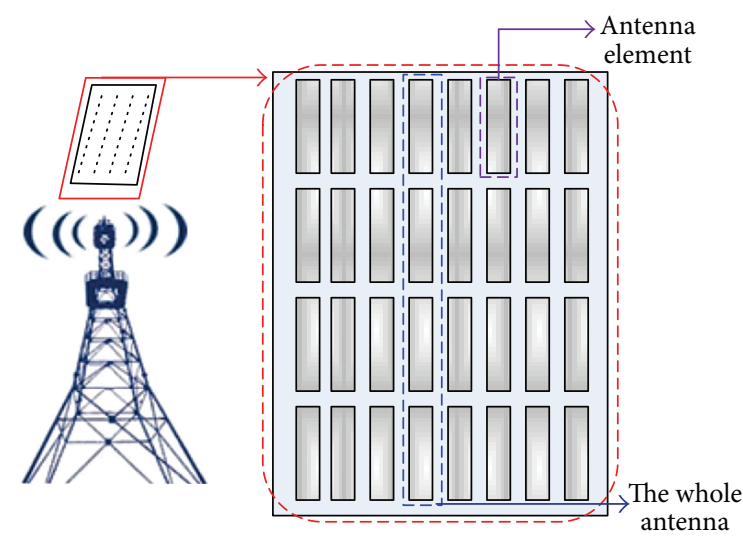

FIGURE 2: Structure of $8 \times 4$ rectangular 3D antenna array.

and receiving antenna remains as a conventional 2D antenna without a different element in vertical dimension. Thus, $\mathbf{H}_{m}^{(k)}$ is the $N_{r} \times\left(N_{t} \times N_{v}\right)$ channel matrix from BS $m$ to UE $k$, and $\mathbf{W}_{m}^{(k)}$ is the $\left(N_{t} \times N_{v}\right) \times l_{k}$ precoding matrix. $\mathbf{n}_{m}^{(k)}$ is the additive white Gaussian noise with zero mean and variance $\mathbb{E}\left(\mathbf{n}_{m}^{(k)} \mathbf{n}_{m}^{(k) H}\right)=\sigma^{2}$. The detailed analysis of detected SINR for the received signal is in $[16,17]$.

2.2. 3D Antenna Modeling. Most geometry-based stochastic radio channel models are two-dimensional (2D) in the sense that they use only geometrical $x y$-coordinates or equivalent parameters of distance and rotation angle. This has been sufficient until these days, while vertical dimension of the arrays and the height of the BS are fully considered in 3D antenna $[18,19]$. For instance, structure of $8 \times 4$ arrays with "rectangular" elements is shown in Figure 2. 


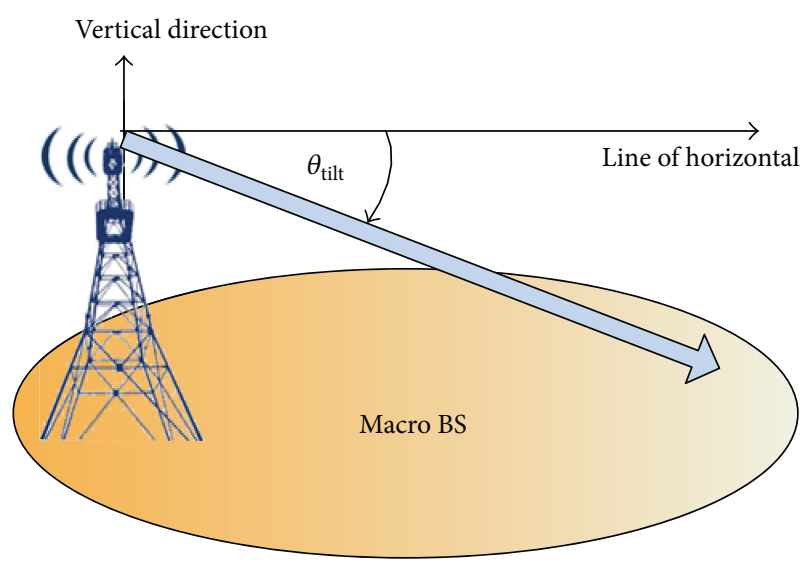

Figure 3: Electrical downtilt.

The whole antenna can be divided into several elements which are controlled by different antenna port. Assuming that $14 \mathrm{~dB}$ is the maximum direction gain of an antenna, equal power is given for each element as defined in

$$
G_{\max }=14-10 \log _{10} N_{v},
$$

where $G_{\max }$ is the maximum directional gain of the array elements and $N_{v}$ is the number of elevation element.

In 3GPP LTE-Advanced simulations, we apply two formulas below for horizontal and vertical radiation patterns so that horizontal antenna gain and vertical antenna gain can be obtained, respectively:

$$
\begin{gathered}
A_{H}(\varphi)=-\min \left[12\left(\frac{\varphi}{\varphi_{3 \mathrm{~dB}}}\right), A_{m}\right], \\
A_{m}=20 \mathrm{~dB}, \\
A_{V}(\theta)=-\min \left[12\left(\frac{\theta-\theta_{\mathrm{tilt}}}{\theta_{3 \mathrm{~dB}}}\right), \mathrm{SLA}_{v}\right], \\
\mathrm{SLA}_{v}=20 \mathrm{~dB},
\end{gathered}
$$

where $\varphi$ is defined as the angle between the direction of interest and the boresight of the antenna in horizontal dimension, which is similar to $\theta$ in vertical dimension. $\varphi_{3 \mathrm{~dB}}$ and $\theta_{3 \mathrm{~dB}}$ are the $3 \mathrm{~dB}$ beamwidth of the horizontal beam and the vertical beam, respectively. $\theta_{\text {tilt }}$ is the downtilt angle in transmitter. $A_{m}$ is the front-to-back attenuation, and SLA $v$ is side lobe attenuation.

The 3D antenna gain is combined as a sum of horizontal pattern and elevation pattern antenna gain. Generation of the $3 \mathrm{D}$ pattern from two perpendicular cross-sections azimuth and elevation patterns is denoted as below:

$$
A(\varphi, \theta)=-\min \left\{-\left[A_{H}(\varphi)+A_{V}(\theta)\right], A_{m}\right\},
$$

The angle of the main beam of the antenna up/below the horizontal plane is called antenna tilt as shown in Figure 3. Positive and negative angles are referred to as downtilt and uptilt, respectively. In electrical downtilt, main, side, and back lobes are tilted uniformly by adjusting phases of antenna elements [20].

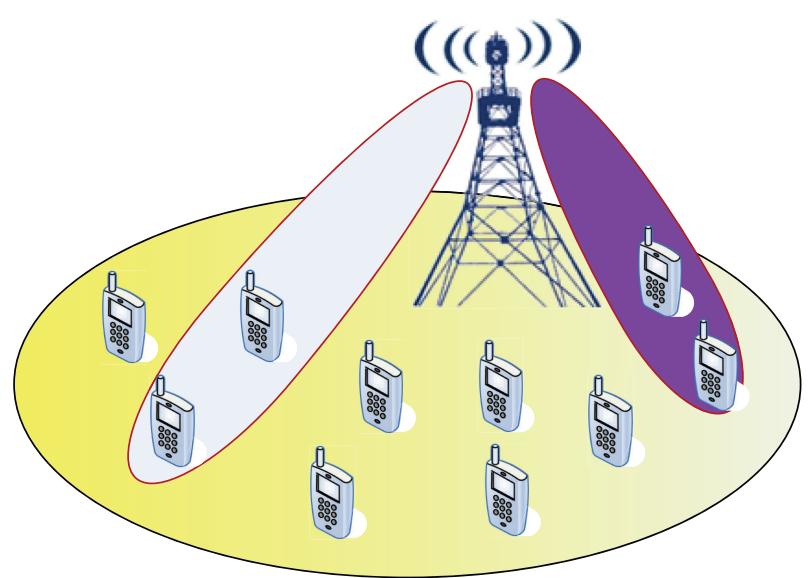

FIGURE 4: Conventional 2D MIMO beamforming.

Angle $\theta_{(i, k)}$ between horizontal line and LOS direction of connecting BS $i$ and UE $k$ in vertical plane is obtained by

$$
\theta_{(i, k)}=\arctan \left(\frac{h_{(i, k)}}{d_{(i, k)}}\right),
$$

where $d_{(i, k)}$ is distance between UE $k$ and BS $i$ and $h_{(i, k)}$ is the height difference between the antenna of UE $k$ and the antenna of BS $i$.

\section{Algorithms for 3D Dynamic Beamforming}

3.1. 2D MIMO Beamforming. Conventional beamforming can be seen as a sort of $2 \mathrm{D}$ cell-specific beamforming. The UE data to be transmitted is processed in accordance with the channel information only in the horizontal dimension. The transmitter forms a small beam within the antenna radiation pattern to point at the UE to be served. This means that only in the horizontal dimension the UE channel state information is being tracked and utilized. Only in the case of several UEs within a cell, the horizontal antenna beam generated by beamforming can distinguish different UEs well.

However, as the UEs in a cell increase substantially, several UEs that request for service at the same time may be located in the same azimuth angle as shown in Figure 4, which means that $2 \mathrm{D}$ antenna gain from the serving $\mathrm{BS}$ is equivalent. Once several UEs are close to each other and located in the cell edge area, they own the same power of desired signal but will suffer serious intercell interference. In this case, transmitting beam simply formed by horizontal dimension beamforming is not enough to distinguish different UEs. For instance, if the beam is aligned with the UE near the base station, the remote UEs will be outside the range of radiation; otherwise, the beam aligned with the UEs far from the base station will result in severe intercell interference to the neighbor cell.

3.2. Proposed Beamforming Algorithm. In the same frequency resource allocation networks, the intercell interference decreases the performance of cell edge UE dramatically. 


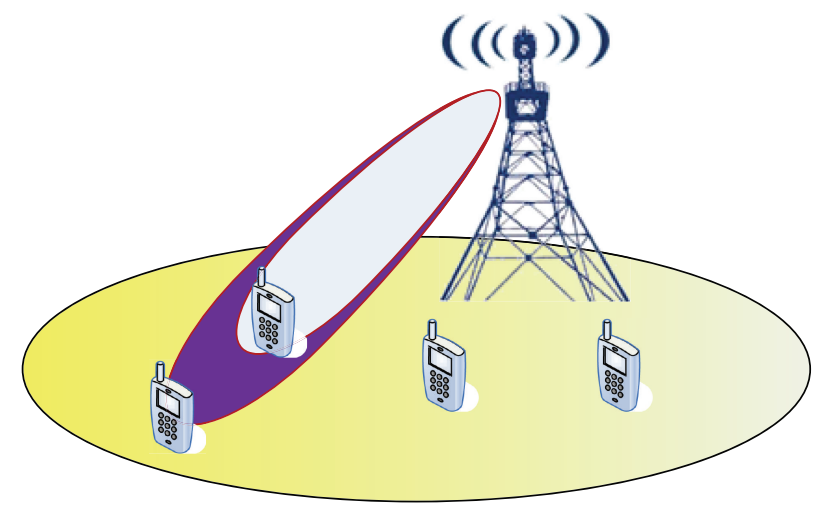

FIGURE 5: 3D dynamic beamforming in horizontal sight.

3D MIMO can be seen as a dynamic interference cancellation method, which aims to eliminate intercell interference coming from neighbor cells and improve the throughput of cell edge UE through 3D dynamic beamforming. For each $\mathrm{UE}$, since the introduction of vertical dimension, difference in height between BS and UE has been taken into account, and UE's position should be considered in three-dimension. According to both horizontal and vertical directions of the specific UE, transmitting beam generated by $3 \mathrm{D}$ antennas can be divided into both horizontal and vertical dimensions, which means it has much less influence on other UEs.

For instance, the two UEs in Figures 5 and 6 have the same azimuth angle. When they are scheduled at the same time, identical horizontal beam direction is shared. Due to the introduction of dynamic beamforming algorithm, transmitter is able to form two different vertical beams through setting different vertical power weight on the antenna elements. Thus, different maximum beam direction can be discriminated for the two specific UEs. Among noncodebook-based precoding technique, the calculation of the 3D MIMO beamforming vector seriously affects the performance of the interference cancellation, and the method of combining suitable horizontal beamforming vector and vertical beamforming vector together should be a big problem needed to be solved.

To implement precoding at BS, an improved beamforming algorithm is adopted. It is assumed that one UE is served by all the antenna elements, and data from a BS is transmitted in a single stream. Antenna number at Macro BS is $N_{t}$, and UE has $N_{r}$ antennas. Firstly, compared with the conventional algorithms such as SVD algorithm and BD algorithm, equivalent process is done with the channel between BSs and UEs, and then traditional precoding methods are utilized. The main procedure of dynamic beamforming is as follows.

Step 1. Computation of horizontal beamforming vector. The horizontal beamforming vector represents the transmitting weight of the elements from different antenna in horizontal dimension. Therefore, the dimension of each horizontal beamforming vector is $N_{t} \times 1$. For there are $N_{v}$ elements in an antenna, the total number of horizontal beamforming vector

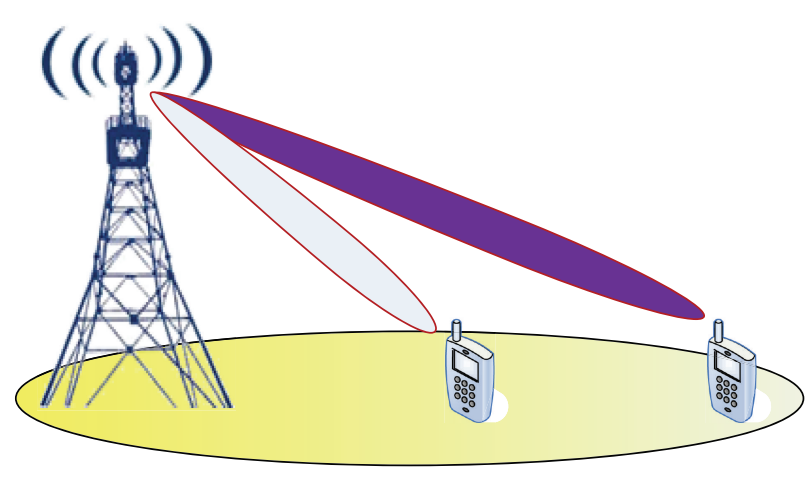

FIGURE 6: 3D dynamic beamforming in vertical sight.

should be $N_{v}$. The channel from $N_{v}$ layer antenna elements in transmitter to $\mathrm{UE}_{k}$ is $\mathbf{H}_{k, 1}, \mathbf{H}_{k, 2}, \ldots, \mathbf{H}_{k, N_{v}}$, respectively, and $\mathbf{H}_{k, i}$ denotes the $N_{r} \times N_{t}$ channel matrix from the $i$ th layer transmitting antenna elements to $\mathrm{UE}_{k} \cdot \mathbf{V}_{k, i} \sim \operatorname{SVD}\left(\mathbf{H}_{k, i}\right) ; \mathbf{V}_{k, i}$ is the $\mathbf{V}$ matrix from the SVD decomposition of $\mathbf{H}_{k, i} \cdot \mathbf{v}_{k, i}$ is the first column of matrix $\mathbf{V}_{i, k}$. The horizontal beamforming vector $\mathbf{v}_{\text {horizontal }}$ for the whole antenna elements can be expressed as

$$
\mathbf{v}_{\text {horizontal }}=\left[\mathbf{v}_{k, 1}^{T}, \mathbf{v}_{k, 2}^{T}, \ldots, \mathbf{v}_{k, N_{v}}^{T}\right],
$$

Step 2. Computation of vertical beamforming vector. The vertical beamforming vector represents the transmitting weight of the elements from a single antenna in vertical dimension. Therefore, the dimension of each vertical beamforming vector is $N_{v} \times 1$. For there is actual $N_{t}$ antenna in transmitter, the total number of vertical beamforming vector should be $N_{t}$. The channel from vertical elements of $N_{t}$ antennas in transmitter to $\mathrm{UE}_{k}$ is $\mathbf{H}_{k, 1}^{*}, \mathbf{H}_{k, 2}^{*}, \ldots, \mathbf{H}_{k, N_{t}}^{*}$, respectively; $\mathbf{H}_{k, i}^{*}$ denotes the $N_{r} \times N_{v}$ channel matrix from the vertical elements of the $i$ th transmitting antenna to $\mathrm{UE}_{k}$. $\mathbf{V}_{k, i}^{*} \sim \operatorname{SVD}\left(\mathbf{H}_{k, i}^{*}\right)$, where $\mathbf{V}_{k, i}^{*}$ is the $\mathbf{V}$ matrix from the SVD decomposition of $\mathbf{H}_{k, i}^{*}$. $\mathbf{v}_{k, i}^{*}$ is the first column of matrix $\mathbf{V}_{k, i}^{*}$. The vertical beamforming vector $\mathbf{v}_{\text {vertical }}$ for the whole antenna elements can be expressed as

$$
\mathbf{v}_{\text {vertical }}=\left[\left(\mathbf{v}_{k, 1}^{*}\right)^{T},\left(\mathbf{v}_{k, 2}^{*}\right)^{T}, \ldots,\left(\mathbf{v}_{k, N_{t}}^{*}\right)^{T}\right],
$$

Step 3. Combination of horizontal beamforming vector and vertical beamforming vector. There are totally $N_{v} \times N_{t}$ antenna elements in transmitter. $\mathbf{H}$ is a combined channel and $\mathbf{H}=\left[\mathbf{H}_{k, 1}^{T}, \mathbf{H}_{k, 2}^{T}, \ldots, \mathbf{H}_{k, N_{v}}^{T}\right]$. Each element has its own horizontal transmitting weight as computed in Step 1. Thus, the complete horizontal beamforming vector's dimension is $N_{v} \times N_{t} \times 1$, combined by $N_{v}$ subvectors which own dimension of $N_{t} \times 1$. Similar process is done with the complete vertical beamforming vector that $N_{t}$ subvectors represent $N_{t}$ antennas' vertical transmitting weight, respectively. Thus, vertical beamforming vector is generated from row combination of subvector $\mathbf{v}_{k, 1}^{*}, \ldots, \mathbf{v}_{k, N_{t}}^{*}$ in Step 2. Finally, the dynamic beamforming vector is obtained from the complete horizontal beamforming vector and the complete vertical 


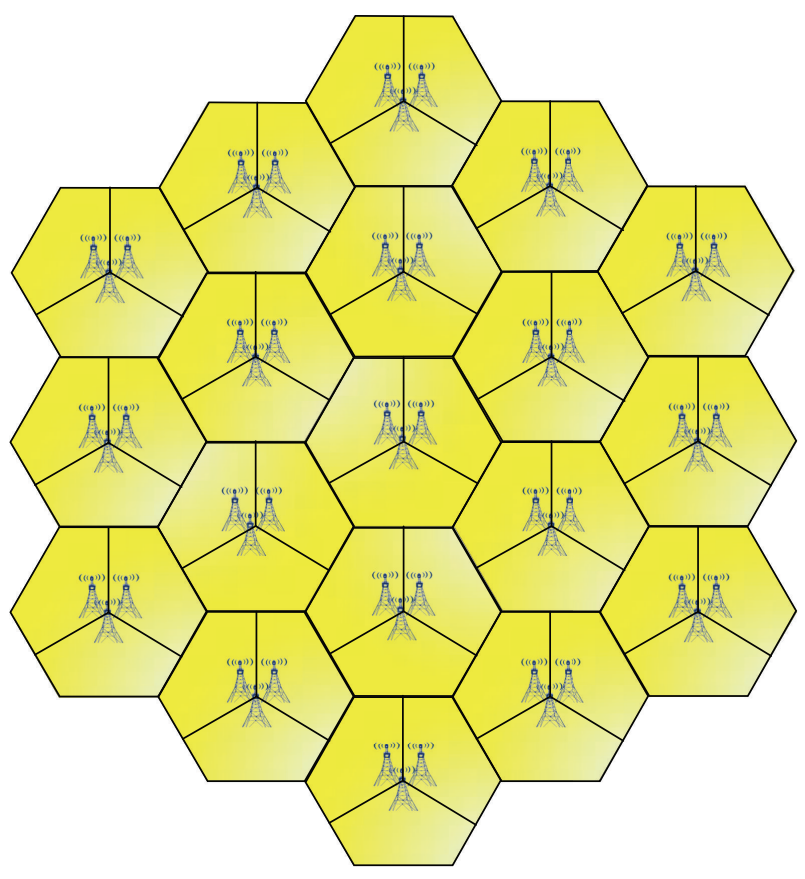

FIgURE 7: 19 cells' topological structure.

beamforming vector, for dot production will be done with these two $N_{v} \times N_{t} \times 1$ vectors. 3D beamforming vector $\mathbf{w}$ is available as follows:

$$
\mathbf{w}=\mathbf{v}_{\text {horizontal }} * \mathbf{v}_{\text {vertical }} \text {, }
$$

where the dimension of $3 \mathrm{D}$ dynamic beamforming vector is $N_{v} \times N_{t} \times 1$, representing $N_{v} \times N_{t}$ antenna elements' dynamic power transmitting weight.

\section{Simulation Results}

In this section, system level performance of the proposed $3 \mathrm{D}$ dynamic beamforming algorithm is evaluated in terms of average spectral efficiency, cell edge UE spectral efficiency, and SINR of the UEs. Consider a system with 19 homogeneous cells as shown in Figure 7. Each cell consists of 3 Macro BSs, which is supposed to provide service to its own sector's UEs. It is worth noting that the $3 \mathrm{BS}$ are located in the center of the cell, which means they share the same coordinate point. In order to eliminate border effect, wrap-around technique is used to simulate interference generated by UEs from at least 2 layers' neighbor cells. Since the simulated scene is configured to Uma (Urban Macro-cell), only outdoor UEs exist during the simulation process. Other detailed simulation parameters are listed in Table 1.

Figure $8(a)$ is the average spectral efficiency of different downtilt angle deployments with sector radius of $288 \mathrm{~m}$. The performance of 3D MIMO without dynamic beamforming has $5 \%, 14 \%, 22 \%$, and $24 \%$ enhancement compared to 2D MIMO networks, respectively. Although array gain due to the introduction of 3D MIMO can be obtained in the system, beamforming gain is not included in the performance
TABLE 1: Simulation configuration parameters.

\begin{tabular}{|c|c|}
\hline Parameters & Assumptions \\
\hline Cell type & Macro cell \\
\hline Cellular layout & 19 cells, each with 3 sectors \\
\hline Sector radius & $288 \mathrm{~m}, 500 \mathrm{~m}$ \\
\hline Bandwidth & $5 \mathrm{M}$ \\
\hline Channel model & WINNER II \\
\hline Shadowing standard deviation & $8 \mathrm{~dB}$ \\
\hline Antenna number & Transmitter: $2 \mathrm{Tx}$, Receiver: $2 \mathrm{Rx}$ \\
\hline Antenna configuration & Co-polarized linear array \\
\hline Antenna spacing & Transmitter: $0.5 \lambda$, Receiver: $0.5 \lambda$ \\
\hline Number of elevation element & 2 \\
\hline Regular antenna gain & $14 \mathrm{dBi}$ \\
\hline Downtilt angle & $8^{\circ}, 10^{\circ}, 12^{\circ}, 14^{\circ}$ \\
\hline BS max TX power & $46 \mathrm{dBm}$ \\
\hline BS height & $25 \mathrm{~m}$ \\
\hline UE height & $1.5 \mathrm{~m}$ \\
\hline UE distribution & Uniformly distributed \\
\hline UE numbers & 10 UEs per sector \\
\hline UE speed & $3 \mathrm{~km} / \mathrm{h}$ \\
\hline Initial UE access criterion & Access by location \\
\hline Scheduler & Proportional fair \\
\hline CQI reporting interval & $5 \mathrm{~ms}$ \\
\hline Delay for scheduling and AMC & $5 \mathrm{~ms}$ \\
\hline Receiver & MMSE \\
\hline Traffic model & Full buffer \\
\hline
\end{tabular}

improvement. As can be seen in the figure, the proposed dynamic beamforming algorithm is able to offer substantial beamforming gain compared to static beamforming. About $14 \%, 24 \%, 32 \%$, and $34 \%$ of the gain compared to $2 \mathrm{D}$ MIMO can be achieved by the novel beamforming algorithm with different downtilt angle parameter. It can be concluded that the performance of $14^{\circ}$ outperforms the performance of other 3 downtilt angles for the scenario with sector radius of $288 \mathrm{~m}$.

Figure $8(\mathrm{~b})$ is the cell edge UE (5\% CDF) spectral efficiency. The proposed dynamic beamforming algorithm offers large performance gain compared to 2D MIMO networks as the intercell interference decreases apparently. For cell edge UEs, the UE spectral efficiency has been increased by about $21 \%, 51 \%, 79 \%$, and $92 \%$, respectively, and the performance of coverage has been increased dramatically.

For different scenarios with sector radius of $500 \mathrm{~m}$, Figure 8 (c) shows the average spectral efficiency of different 


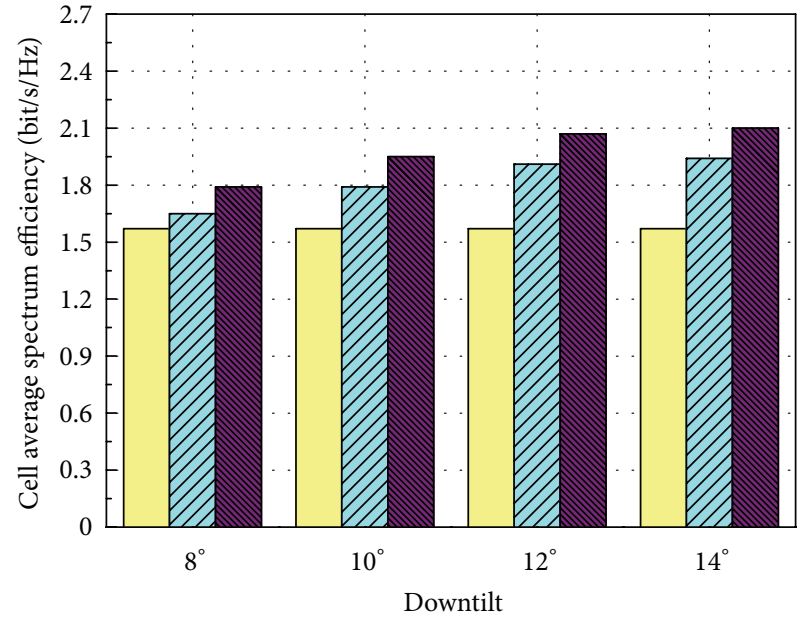

(a)

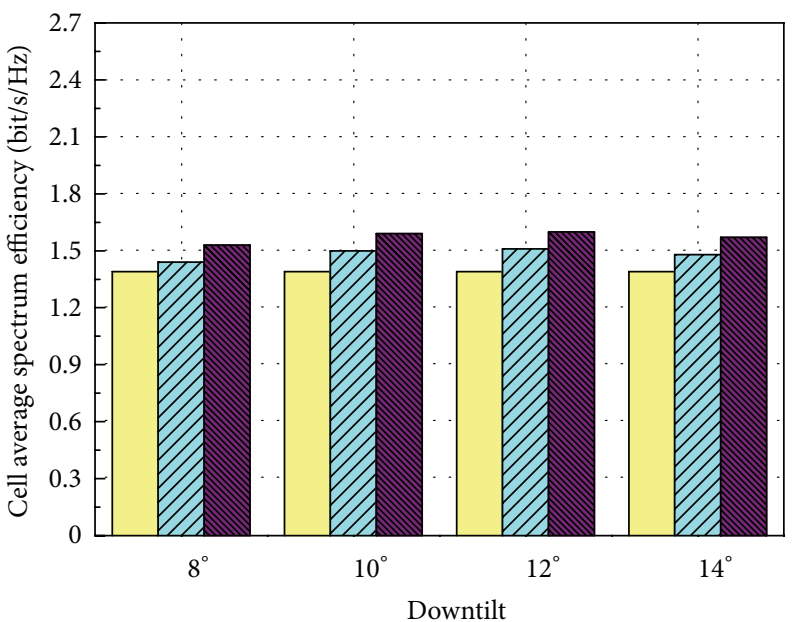

2D MIMO

3D MIMO without dynamic beamforming 3D MIMO with dynamic beamforming

(c)

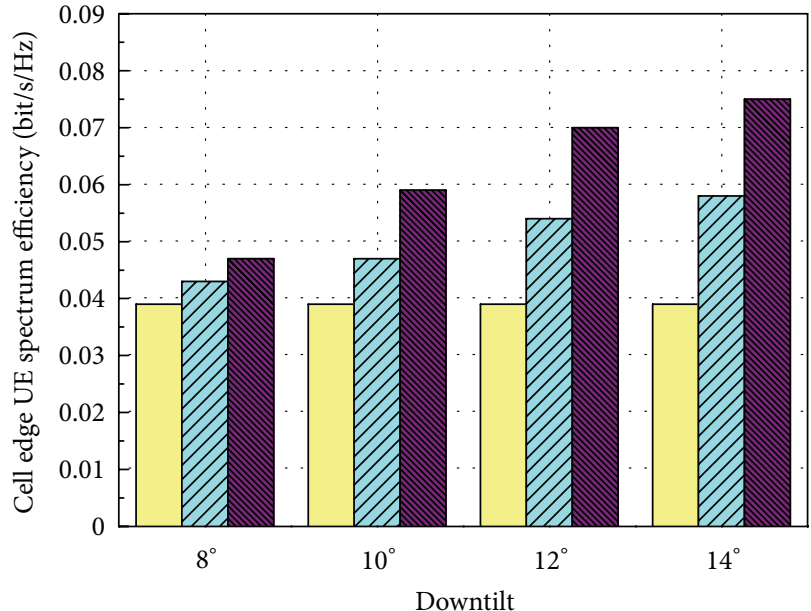

(b)

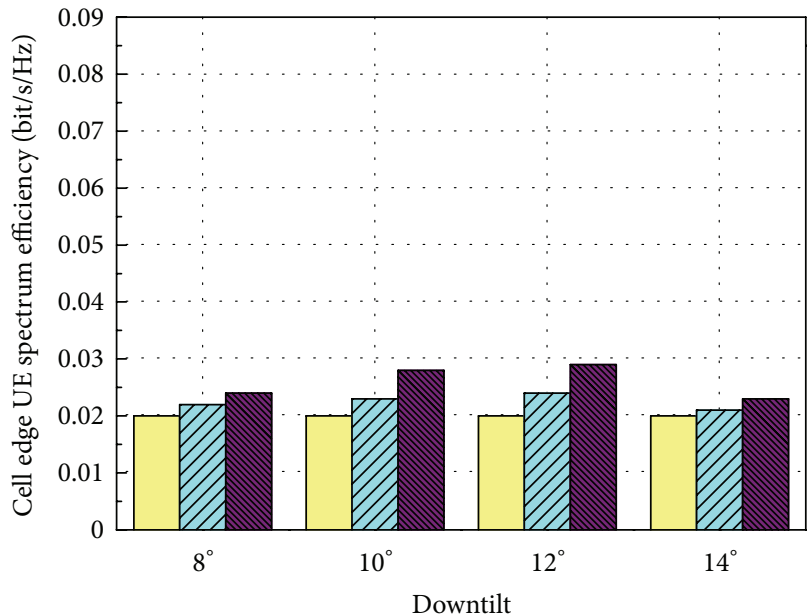

$2 \mathrm{D}$ MIMO

3D MIMO without dynamic beamforming 3D MIMO with dynamic beamforming

(d)

FIGURE 8: System level results of 2D MIMO, 3D MIMO without dynamic beamforming and 3D MIMO with dynamic beamforming: (a) cell average spectral efficiency with sector radius of $288 \mathrm{~m}$; (b) cell edge UE spectral efficiency with sector radius of $288 \mathrm{~m}$; (c) cell average spectral efficiency with sector radius of $500 \mathrm{~m}$; (d) cell edge UE spectral efficiency with sector radius of $500 \mathrm{~m}$.

downtilt angle deployments, and the performance of $3 \mathrm{D}$ MIMO with dynamic beamforming has $10 \%, 14 \%, 15 \%$, and $13 \%$ enhancement compared to $2 \mathrm{D}$ MIMO networks, respectively. Since the radius of sector increases, the distribution of UEs within a sector changes a lot, and the elevation angles of UEs in the area decrease. Downtilt angle should be adjusted so as to cover more UEs. It can be observed from Figure 8 that the performance of $12^{\circ}$ outperforms the performance of other 3 downtilt angles for the scenario with sector radius of $500 \mathrm{~m}$.
For cell edge UEs, the UE spectral efficiency has been increased by about $20 \%, 40 \%, 45 \%$, and $15 \%$ respectively as presented in Figure 8(d). Although the density of UE changed, the proposed algorithm can still offer considerable gain compared with 2D MIMO networks.

Figure 9 shows the receive SINR CDF curves of 2D MIMO UEs, 3D MIMO without dynamic beamforming UEs, and 3D MIMO with dynamic beamforming UEs. For scenario with sector radius of $288 \mathrm{~m}$, the downtilt angle is set for $14^{\circ}$ as summarized above. With the proposed algorithm, 


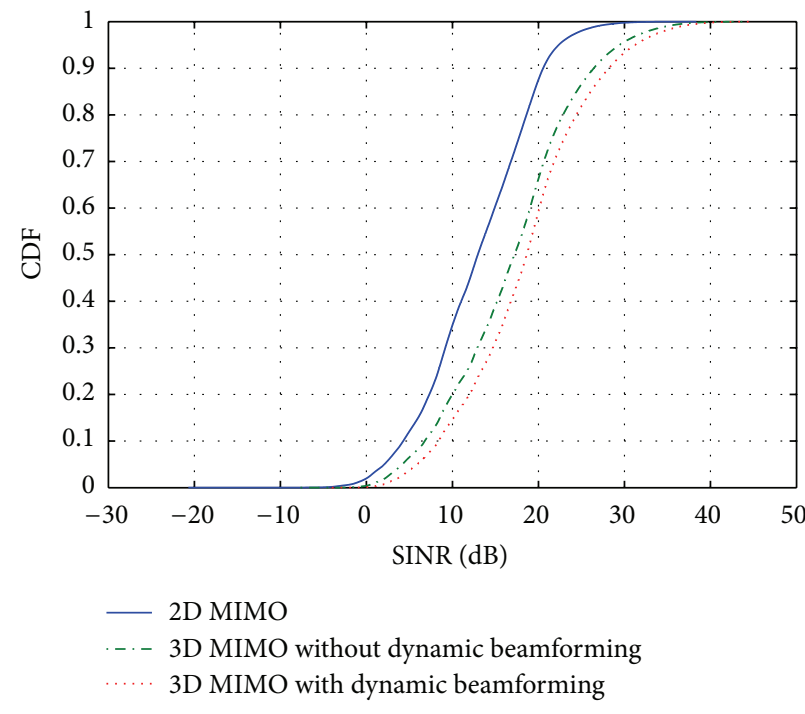

(a)

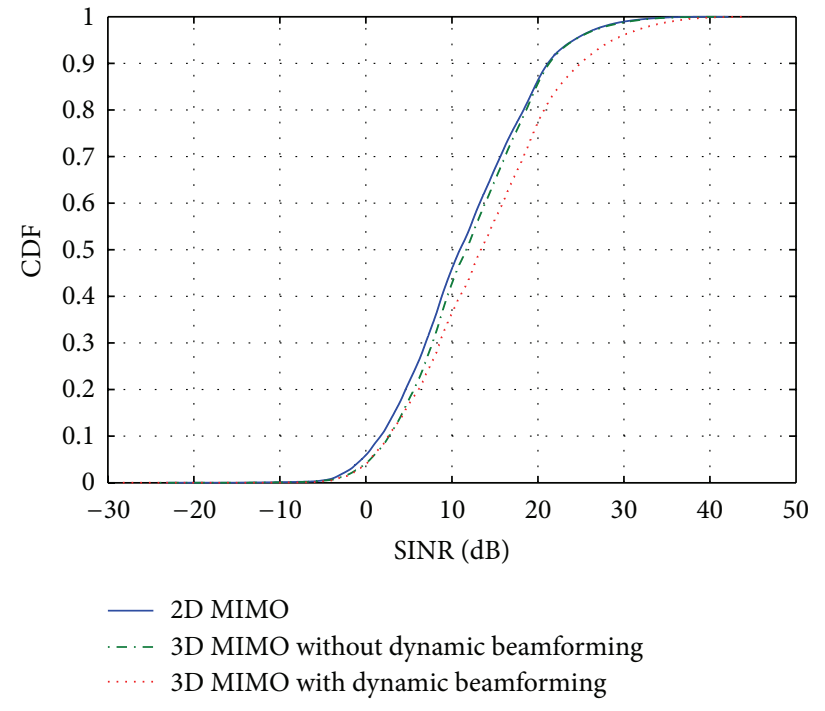

(b)

FIGURE 9: System level results of receive SINR CDF for 2D MIMO, 3D MIMO without dynamic beamforming and 3D MIMO with dynamic beamforming: (a) sector radius: $288 \mathrm{~m}$; (b) sector radius: $500 \mathrm{~m}$.

the SINR of the 3D MIMO with dynamic beamforming outperforms by $6 \mathrm{~dB}$ compared to the 2D MIMO and has approximately $2 \mathrm{~dB}$ gain compared with the $3 \mathrm{D}$ MIMO without dynamic beamforming. Both cell average UE and cell edge UE can be served much better than before.

Under the influence of larger sector radius, the change of downtilt configuration is not so apparent as it performs in $288 \mathrm{~m}$ scenario. Compared with the UEs in $288 \mathrm{~m}$ scenario, some UEs in $500 \mathrm{~m}$ scenario will receive decreased signal power so that less gain can be obtained in the average received SINR of UEs in $500 \mathrm{~m}$ scenario. Thus, there is not much difference between the SINR curved line of 2D MIMO UE and the SINR curved line of 3D MIMO UE as depicted in Figure 9(b). In spite of this, the positive effect brought by the proposed algorithm is still obvious.

\section{Conclusion}

In this paper, modeling of $3 \mathrm{D}$ channel and structure of $3 \mathrm{D}$ antenna have been introduced. A dynamic beamforming algorithm for 3D MIMO in LTE-Advanced networks is proposed, which can be proved as an effective method to reduce intercell interference. Simulation results for 3D MIMO with different downtilt angle parameters and sector radius parameters are presented. Compared with $2 \mathrm{D}$ beamforming, 3D dynamic beamforming can improve both the cell edge UE throughput and the whole system's performance significantly. At most 34\% gain for cell center UE and $92 \%$ gain for cell edge UE over the conventional 2D beamforming are achieved, and the performance differences of different scenarios are analyzed in this paper.

\section{Acknowledgments}

This work was supported in part by the State Major Science and Technology Special Projects (Grant no. 2012ZX03001028004, 2013ZX03001001-002, and 2013ZX03001001-003) and the Beijing Natural Science Foundation (Grant no. 4131003).

\section{References}

[1] M. Peng and W. Wang, "Technologies and standards for TDSCDMA evolutions to IMT-advanced," IEEE Communications Magazine, vol. 47, no. 12, pp. 50-58, 2009.

[2] M. Peng, W. Wang, and H.-H. Chen, "TD-SCDMA evolution," IEEE Vehicular Technology Magazine, vol. 5, no. 2, pp. 28-41, 2010.

[3] 3GPP TS 36. 814 V0. 4. 1, Evolved universal terrestrial radio access (E-UTRA), Further advancements for E-UTRA physical layer aspects.

[4] L. Liu, R. Chen, S. Geirhofer, K. Sayana, Z. Shi, and Y. Zhou, "Downlink MIMO in LTE-advanced: SU-MIMO vs. MUMIMO," IEEE Communications Magazine, vol. 50, no. 2, pp. 140-147, 2012.

[5] M. Peng, Z. Ding, Y. Zhou, and Y. Li, "Advanced self-organizing technologies over distributed wireless networks," International Journal of Distributed Sensor Networks, vol. 2012, Article ID 821982, 2 pages, 2012.

[6] O. N. C. Yilmaz, S. Hämäläinen, and J. Hämäläinen, "Analysis of antenna parameter optimization space for 3GPP LTE," in Proceedings of the IEEE 70th Vehicular Technology Conference Fall (VTC '09), Anchorage, Alaska, USA, September 2009.

[7] S. K. Mohammed, A. Zaki, A. Chockalingam, and B. S. Rajan, "High-rate space-time coded large-MIMO systems: lowcomplexity detection and channel estimation," IEEE Journal on 
Selected Topics in Signal Processing, vol. 3, no. 6, pp. 958-974, 2009.

[8] M. Peng, G. Xu, W. Wang, and H.-H. Chen, "McWiLL-a new mobile broadband access technology for supporting both voice and packet services," IEEE Systems Journal, vol. 4, no. 4, pp. 495504, 2010.

[9] F. Rusek, D. Persson, B. K. Lau et al., "Scaling up MIMO: opportunities and challenges with very large arrays," IEEE Signal Processing Magazine, vol. 30, no. 1, 2013.

[10] P. Zetterberg, "Performance of antenna tilting and beamforming in an urban macrocell," in Proceedings of the 1st International Conference on Wireless Communication, Vehicular Technology, Information Theory and Aerospace and Electronic Systems Technology, Wireless (VITAE '09), pp. 201-206, Aalborg, Denmark, May 2009.

[11] IST-4-027756 WINNER II D1.1.2 V1.2 WINNER II channel models.

[12] IST-WINNER D5.3 WINNER+ Final channel models v1.0.

[13] IST-2003-507581 WINNER D5.4 Final report on link level and system level channel models v1.4.

[14] X. You, D. Wang, P. Zhu, and B. Sheng, "Cell edge performance of cellular mobile systems," IEEE Journal on Selected Areas in Communications, vol. 29, no. 6, pp. 1139-1150, 2011.

[15] T. Zhou, M. Peng, W. Wang, and H. Chen, "Low-complexity coordinated beamforming for downlink multi-cell SDMA/OFDM system," IEEE Transactions on Vehicular Technology, vol. 62, no. 1, 2013.

[16] F. Shu, L. Lihua, C. Qimei, and Z. Ping, "Non-unitary codebook based precoding scheme for multi-user MIMO with limited feedback," in Proceedings of the IEEE Wireless Communications and Networking Conference (WCNC '08), pp. 678-682, April 2008.

[17] M. Peng, X. Zhang, W. Wang, and H.-H. Chen, "Performance of dual-polarized MIMO for TD-HSPA evolution systems," IEEE Systems Journal, vol. 5, no. 3, pp. 406-416, 2011.

[18] M.-T. Dao, V.-A. Nguyen, Y.-T. Im, S.-O. Park, and G. Yoon, "3D polarized channel modeling and performance comparison of MIMO antenna configurations with different polarizations," IEEE Transactions on Antennas and Propagation, vol. 59, no. 7, pp. 2672-2682, 2011.

[19] G. Auer, “3D MIMO-OFDM channel estimation," IEEE Transactions on Communications, vol. 60, no. 4, pp. 972-985, 2012.

[20] O. N. C. Yilmaz, S. Hämäläinen, and J. Hämäläinen, "Comparison of remote electrical and mechanical antenna downtilt performance for 3GPP LTE," in Proceedings of the IEEE 70th Vehicular Technology Conference Fall (VTC '09), Anchorage, Alaska, USA, September 2009. 

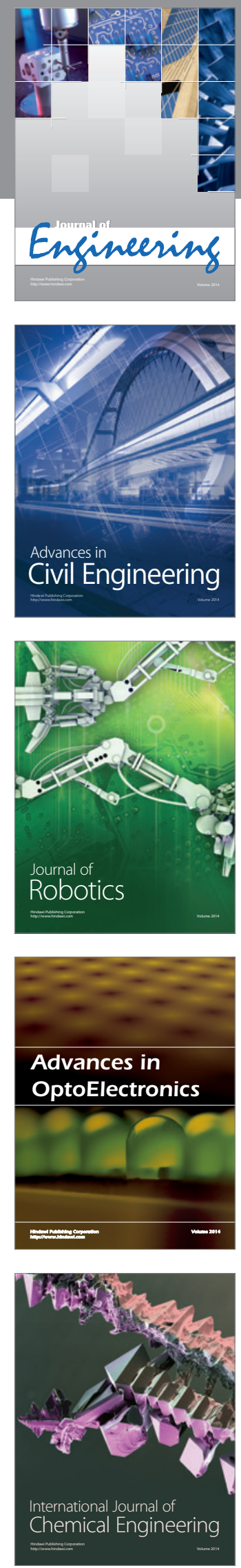

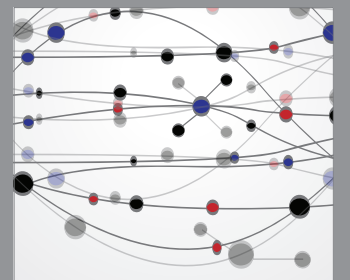

The Scientific World Journal
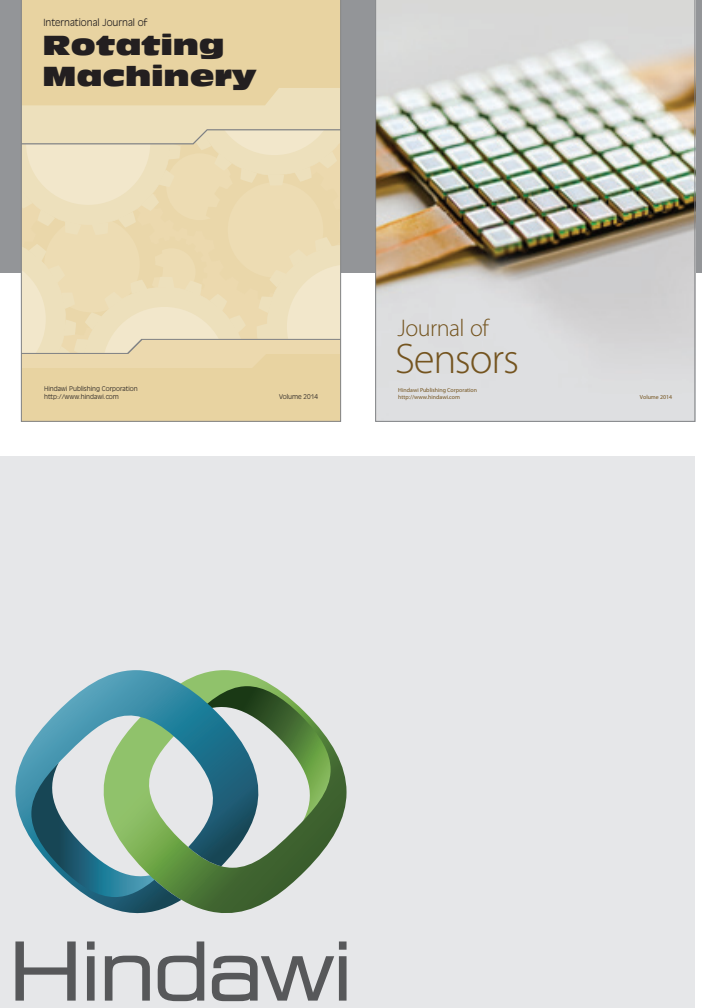

Submit your manuscripts at http://www.hindawi.com
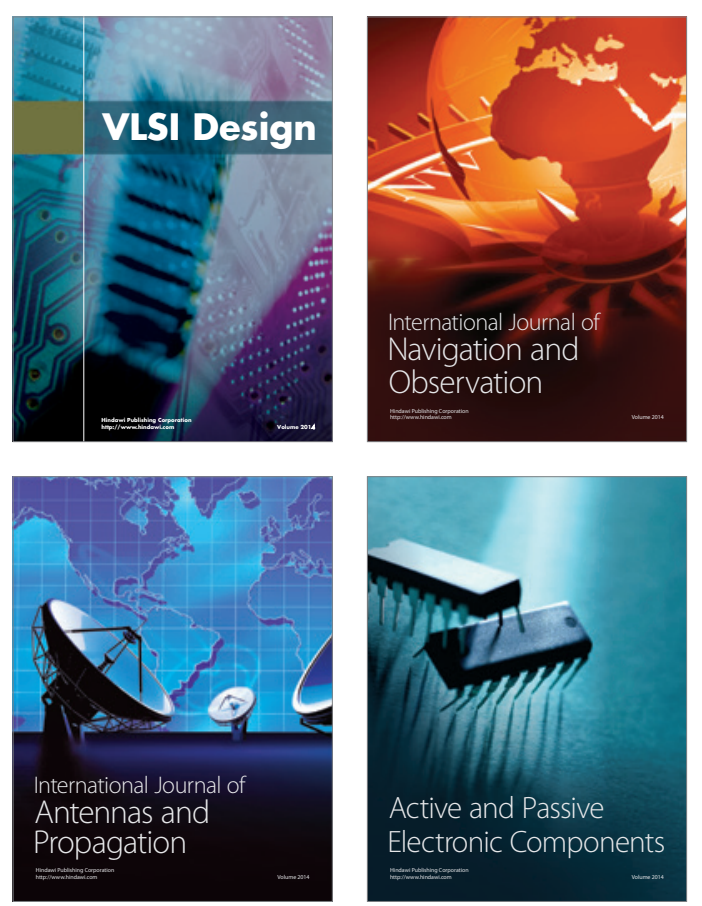
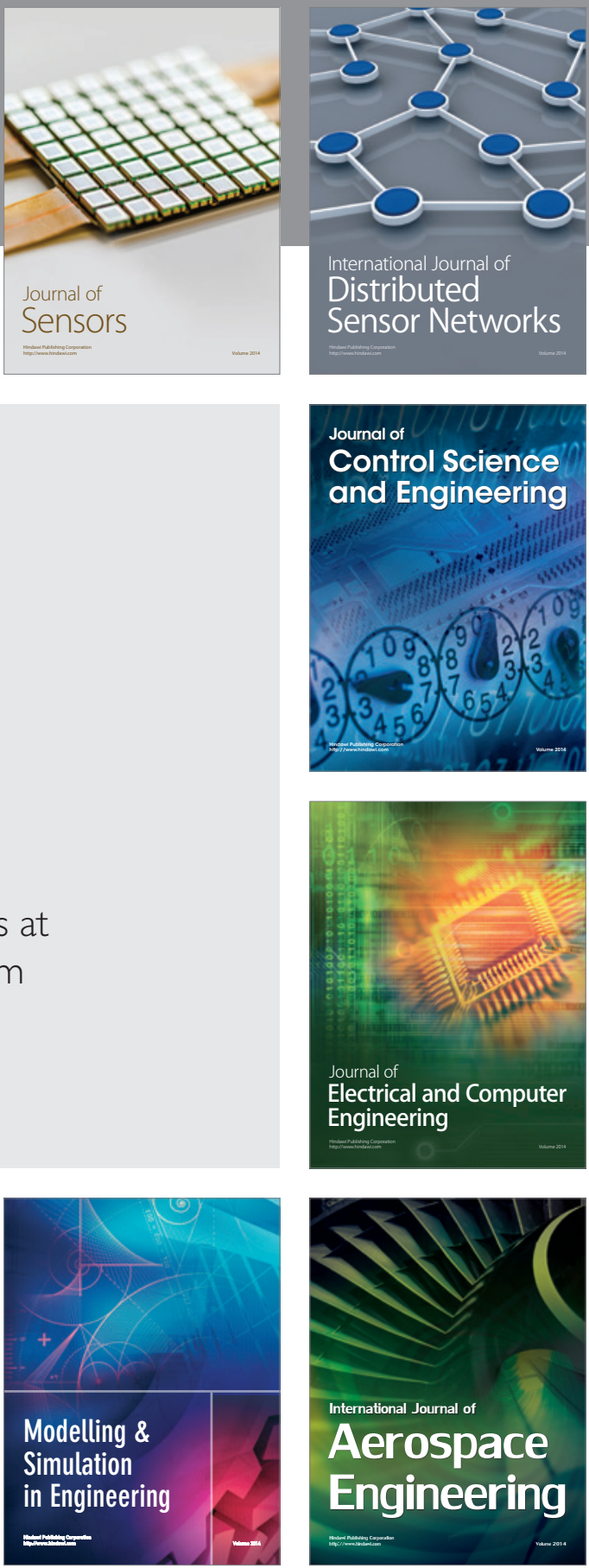

Journal of

Control Science

and Engineering
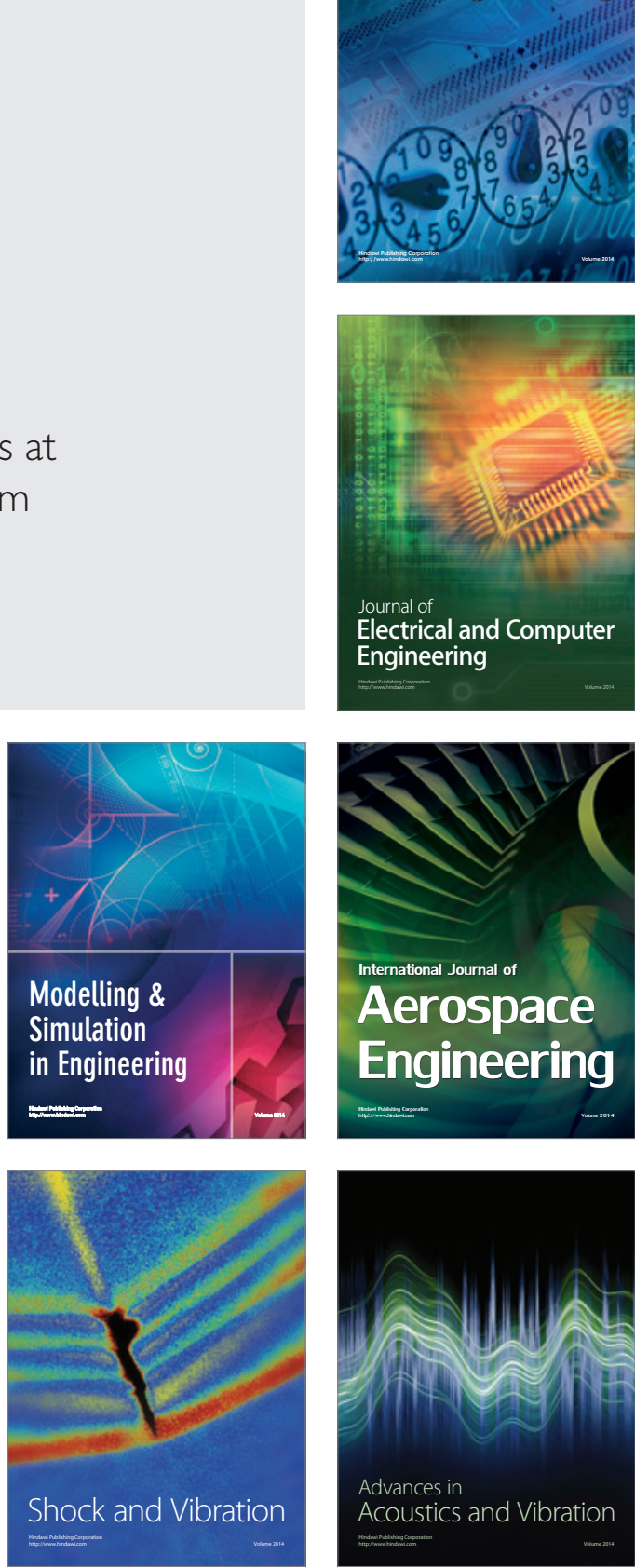